\title{
Theory for the Spatiotemporal Dynamics of Domain Walls close to a Nonequilibrium Ising-Bloch Transition
}

\author{
Damià Gomila, Pere Colet, and Daniel Walgraef \\ IFISC (CSIC-UIB), Instituto de Física Interdisciplinar y Sistemas Complejos, E-07122 Palma de Mallorca, Spain
}

(Received 17 June 2014; published 27 February 2015)

\begin{abstract}
We derive a generic model for the interaction of domain walls close to a nonequilibrium-Bloch transition. The universal scenario predicted by the model includes stationary Ising and Bloch localized structures (dissipative solitons), as well as drifting and oscillating Bloch structures. Our theory also explains the behavior of Bloch walls during a collision. The results are confirmed by numerical simulations of the Ginzburg-Landau equation forced at twice its natural frequency and are in agreement with previous observations in several physical systems.
\end{abstract}

DOI: 10.1103/PhysRevLett.114.084101

PACS numbers: 05.45.-a, 42.65.-k, 75.60.Ch, 82.40.Bj

Domain walls are common coherent structures in bistable extended systems, and their dynamics and interaction determine the different spatiotemporal regimes in a large variety of driven dissipative systems in fields as diverse as chemistry, physics, biology, material science, ecology, or optics. Walls connecting two homogeneous states can be classified according to their symmetry with respect to the wall center as Ising, which are symmetric, and Bloch, which are not. Associated to their asymmetry, Bloch walls have a defined chirality [1]. Transitions between Ising and Bloch domain walls are well known in both equilibrium and nonequilibrium systems [2-6]. They are associated to spontaneous chirality breaking and have been thoroughly studied in different physical systems $[1,5,7,8]$. In nonequilibrium systems, as the result of nonvariational effects, chirality breaking induces the motion of Bloch walls [4]. More recently, it has also been shown that delayed feedback can also induce motion in an Ising-Bloch (IB) transition $[9,10]$.

A crucial ingredient determining the dynamical evolution of domain walls is their interaction. While the interaction between Ising fronts has been widely studied in the literature, leading to the formation of localized structures (LS) or dissipative solitons in a wide range of complex systems [11-16], only specific cases concerning the interaction of Bloch walls have been considered [17-21]. In this Letter we show that the interaction of Bloch walls can be very generally described in terms of only two coupled modes of the front, the neutral (or Goldstone) mode and the chiral mode that become unstable at the IB transition. Our reduced model provides a universal framework to understand the instabilities and dynamical regimes one can observe when two fronts interact in any system close to an Ising-Bloch transition.

We consider the complex Ginzburg Landau equation parametrically forced at twice the natural frequency (PCGLE), which is a paradigm for the study of Ising and Bloch walls in nonequilibrium systems. In fact, recently there has been a new surge of interest in the Ginzburg-Landau equation coming from new applications in nonlinear optics such as frequency comb generation. In particular, it has been shown that ring-cavity configurations [22-24] and delayed systems for which one can identify two time scales [25-28] can be recast to extended systems described by the Ginzburg-Landau equation where the spatial coordinate is associated with the fast time scale while the slow time plays the role of the usual time describing the evolution of the field. This make the results presented in this Letter relevant not only to spatial systems but also to this special kind of temporal system.

The PCGLE writes

$$
\partial_{t} A=(\mu+i \nu) A+\gamma A^{*}-(1+i \beta)|A|^{2} A+(1+i \alpha) \nabla^{2} A
$$

and for $\gamma^{2}\left(1+\beta^{2}\right)>(\nu-\beta \mu)^{2}$ it has two equivalent stable homogeneous steady states $A_{ \pm}$, such that $A_{+}=-A_{-}=\left|A_{+}\right| e^{i \Phi_{0}}$. The existence and stability of Ising $A_{I}(x)$ and Bloch $A_{B}(x)$ walls connecting these two states have been thoroughly studied [4,29-31]. $A_{I, B}(x)$ tend to $A_{ \pm}$for $x \rightarrow-\infty$ and to $A_{\mp}$ for $x \rightarrow \infty$. For an Ising wall centered at $x=x_{w}, A_{I}\left(x_{w}\right)=0$. An IB transition occurs when the Ising wall becomes unstable versus chiral perturbations [4], such that fronts acquire chirality $\left[A\left(x_{w}\right) \neq 0\right]$.

For $\nu=\beta=\alpha=0$ Ising and Bloch walls are [32]

$$
\begin{aligned}
& A_{I}=\sqrt{\mu+\gamma} \tanh \sqrt{(\mu+\gamma) / 2} x \\
& A_{B}=\sqrt{\mu+\gamma} \tanh \sqrt{2 \gamma} x+i \frac{\sqrt{\mu-3 \gamma}}{\cosh \sqrt{2 \gamma} x} .
\end{aligned}
$$

Ising walls are stable for $\gamma \geq(\mu / 3)$. At $\gamma=(\mu / 3)$ Bloch walls appear through a pitchfork bifurcation. For $\gamma<(\mu / 3)$ Bloch walls are stable while Ising walls are unstable. 

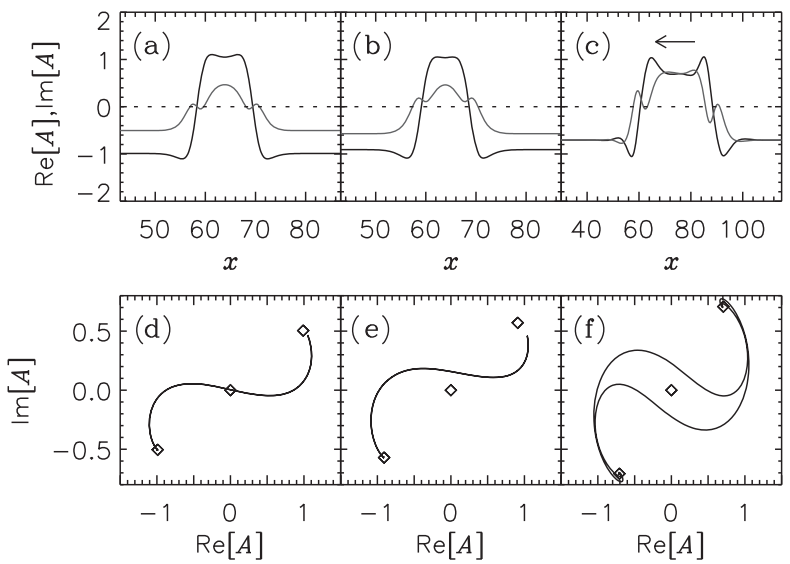

FIG. 1. (a) Ising LS of Eq. (1) for $\gamma=0.4$, (b) resting Bloch LS for $\gamma=0.35$, and (c) drifting Bloch LS for $\gamma=0.3$, above the IB transition. Other parameters: $\mu=1, \nu=0.2, \beta=-0.1$, and $\alpha=1$. Panels (d)-(f) show the homoclinic trajectory in the plane $(\Re[A], \Im[A])$.

For general $\nu \neq 0, \beta \neq 0, \alpha \neq 0$ Eq. (1) still has Ising and Bloch wall solutions, but they have to be computed numerically [4]. Because of the nonvariational terms, Bloch walls move with a velocity proportional to their chirality [1,29-31]. Both Ising and Bloch walls also present spatially oscillatory tails. This affects the interaction between walls leading to a rich dynamical behavior, as explained in this Letter and observed, for instance, in localized current filaments [17], optical cavities [18], or in systems with feedback [33].

Performing numerical simulations of (1) for parameter values around the IB transition, very generally, one observes distinctive dynamical regimes. Well before the IB transition, Ising walls bound due to oscillatory tail interaction forming LS [Fig. 1(a)]. Each side of the Ising LS is essentially an Ising front $\left[A\left(x_{w}\right) \simeq 0\right.$, Fig. 1(d)]. This mechanism for the formation of LS has been largely studied (see, for instance, [34-38]), and commonly observed in different physical systems [11-16].

Beyond the IB transition LS formed by two Bloch walls with opposite chirality [Figs. 1(c) and 1(f)] are observed. Since both walls have opposite orientation the result is that both move in the same direction and the LS drifts. Such a bound state was observed in [18]. Other kinds of LS can also undergo an IB transition leading to drifting LS [39-41].

We also find a different kind of LS formed by two Bloch walls with the same chirality and opposite orientation [Figs. 1(b) and 1(e)]. The two walls push or pull in opposite directions, but the oscillatory tail's interaction keeps them bound and the LS is at rest. These resting Bloch LS occurs for parameters before the IB transition, where isolated walls are always of the Ising type.

For other parameters, still below the IB transition, we observe oscillating LS (Fig. 2) in which chirality induced
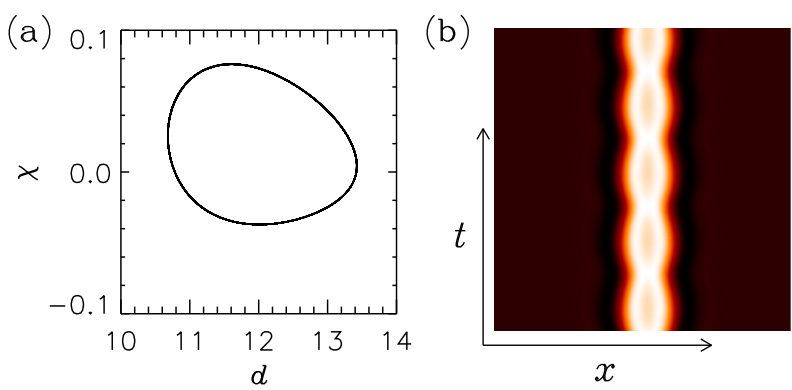

FIG. 2 (color online). Oscillatory Bloch LS. (a) Cycle in the phase space $(\chi, d)$, where the chirality $\chi$ is measured as $\Im[A]$ at the spatial location where $\Re[A]=0$. (b) Spatiotemporal representation of $\Re[A]$. Bright (dark) colors indicate high (low) values. Here $\mu=1, \nu=-0.2, \gamma=0.35, \beta=-0.5$, and $\alpha=2$.

motion manifests but domain walls remain bound. The wall separation $d$ and the chirality $\chi$ oscillate periodically. Such a regime has been observed in localized current filaments [17] and optical cavities [18]. Beyond the IB transition this cycle is destroyed and the domain walls escape to infinity. In this case Bloch walls moving towards each other collide and bounce back (Fig. 3), as also reported in [18].

To understand the different dynamical regimes described above, we study, close to the IB transition, the dynamics of two opposite fronts located at $x_{1}(t)$ and $x_{2}(t)\left(x_{1}<x_{2}\right)$ using the ansatz

$$
\begin{aligned}
\tilde{A}_{\mathrm{LS}}(x, t)= & \tilde{A}_{\mathrm{s}}\left[x-x_{1}(t)\right]-\tilde{A}_{\mathrm{s}}\left[x-x_{2}(t)\right]-\left|A_{+}\right| \\
& +\chi_{1}(t) \tilde{A}_{\mathrm{a}}\left[x-x_{1}(t)\right]+\chi_{2}(t) \tilde{A}_{\mathrm{a}}\left[x-x_{2}(t)\right] \\
& +\epsilon W(x, t)
\end{aligned}
$$

where $\tilde{A} \equiv A e^{-i \Phi_{0}}, \tilde{A}_{\mathrm{s}}\left(x-x_{j}\right)$ is the front symmetric part, $\tilde{A}_{a}\left(x-x_{j}\right)$ the chiral mode becoming unstable at the IB transition and $\chi_{j}(t)$ its amplitude. For small $\nu, \beta$, and $\alpha$, $\tilde{A}_{s} \approx X$ and $\tilde{A}_{a} \approx i Y$, being $X$ and $Y$ the real and imaginary parts of the front. Replacing (3) in (1) and projecting on the neutral modes $X^{\prime}\left(x-x_{j}\right)$ ( refers to the spatial derivative) and chiral modes $Y\left(x-x_{j}\right)$ we get [42]
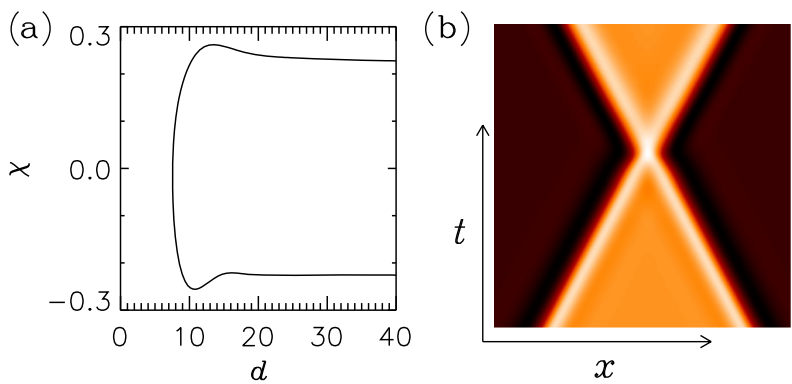

FIG. 3 (color online). Bouncing of two Bloch walls above the IB transition. Here $\gamma=0.3$. Other parameters as in Fig. 2. 


$$
\begin{aligned}
\dot{d} & =f(d)+\kappa\left(\chi_{1}+\chi_{2}\right), \\
\dot{D} & =-\kappa\left(\chi_{1}-\chi_{2}\right), \\
\dot{\chi}_{1} & =g(d)+\eta \chi_{1}-\rho \chi_{1}^{3}, \\
\dot{\chi}_{2} & =g(d)+\eta \chi_{2}-\rho \chi_{2}^{3},
\end{aligned}
$$

The neutral modes of the fronts lead to a global neutral and an interaction mode with amplitudes proportional to $D(t)=x_{1}(t)+x_{2}(t)$ and $d(t)=x_{2}(t)-x_{1}(t)$. In (4)

$$
\begin{aligned}
f(d) & =2 \Gamma_{I} \int_{-\infty}^{+\infty}\left\{\Re\left[R_{-}(x, d)\right] X_{\mathrm{LS}}(x, d)\right\} X^{\prime}(x) d x, \\
g(d) & =\Gamma_{B} \int_{-\infty}^{+\infty}\left\{\Im\left[R_{-}(x, d)\right] X_{\mathrm{LS}}(x, d)\right\} Y(x) d x, \\
\kappa & =\Gamma_{I} \int_{-\infty}^{+\infty}\left\{\left[\Im\left[R_{+}(x, d)\right]-2 \nu\right] Y(x)\right\} X^{\prime}(x) d x, \\
\eta & =\Gamma_{B} \int_{-\infty}^{+\infty}\left\{\left(2 \mu-\Re\left[R_{+}(x, d)\right]\right) Y(x)\right\} Y(x) d x, \\
\rho & =\Gamma_{B} \int_{-\infty}^{+\infty} Y^{4}(x) d x,
\end{aligned}
$$

where $X_{\mathrm{LS}}(x, d)=X(x)-X(x-d)-\left|A_{+}\right|, \quad R_{ \pm}(x, d)=$ $(1+i \beta)\left[\left|A_{+}\right|^{2} \pm X_{\mathrm{LS}}^{2}(x, d)\right] \mp(1+i \alpha) \nabla_{x}^{2}$, and $\Gamma_{I, B}$ such that

$$
\Gamma_{I} \int_{-\infty}^{+\infty} X^{\prime 2}(x) d x=\Gamma_{B} \int_{-\infty}^{+\infty} Y^{2}(x) d x=1
$$

$f(d)$ is the interaction between individual walls and has been found to behave as $f(d) \simeq-b \cos (q d) e^{-r d}$ [32]. Close to the IB transition $g(d)$ is proportional to $f(d)$. $\eta=0$ corresponds to the IB transition.

Equation (4) admits solutions of the form $\chi_{1}=-\chi_{2}=\chi$, for which $D(t)=-2 \kappa \chi t$, while $d$ relaxes to a fixed point given by a zero of $f(d)$, so that the structure moves as a whole. This case was considered in [45] and corresponds to the drifting Bloch LS discussed above [Figs. 1(c) and 1(f)].

Equation (4) also admits solutions of the form $\chi_{1}=$ $\chi_{2}=\chi$ so that the center of mass $D$ does not move and

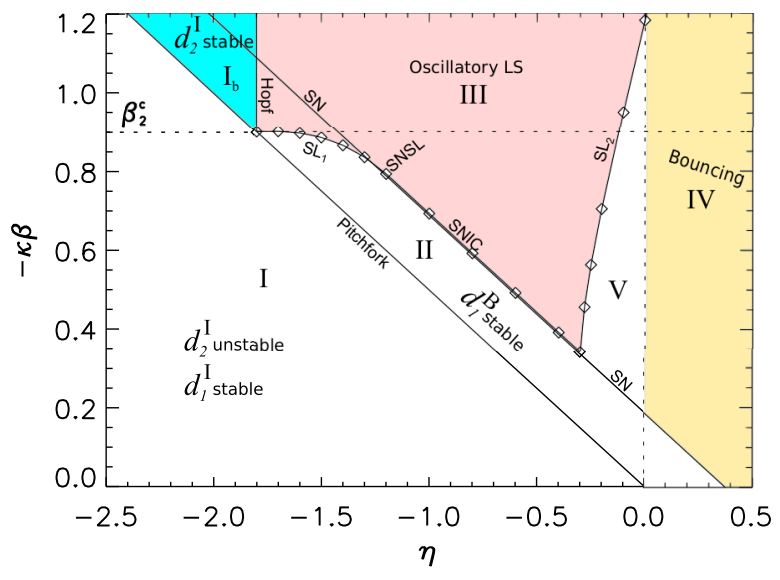

FIG. 4 (color online). Phase diagram of Eq. (7) for $\kappa=0.3$ (see text). We show only the bifurcation lines of the fixed points $d_{n}^{I}$ and $d_{n}^{B}$ with $n=1,2$. The phase diagram is qualitatively the same for other values of $n$.

$$
\begin{aligned}
& \dot{d}=-b \cos (q d) e^{-r d}+2 \kappa \chi, \\
& \dot{\chi}=-\beta b \cos (q d) e^{-r d}+\eta \chi-\rho \chi^{3} .
\end{aligned}
$$

In this case the system presents a rich variety of dynamical regimes (see phase diagram in Fig. 4). One can rescale $\chi, d$ and $t$ so that $\rho=b=r=1$ and, without loss of generality, take $\kappa>0$. We consider negative values of $\beta$, since, as explained later, this is the case describing the scenario observed in the full model (1). The case with positive values of $\beta$ will be reported elsewhere.

Equation (7) have zero chirality fixed points given by

$$
\chi^{I}=0, d_{n}^{I}=(2 n+1) \pi / 2 q, \quad n=0,1,2, \ldots,
$$

which correspond to Ising LS of the full model [Figs. 1(a) and 1(d)] with different widths. A linear stability analysis shows that, for $\eta<2 \kappa \beta$, the fixed points with $n$ odd are stable, while those with $n$ even are saddles (region I in Fig. 4).

Increasing $\eta$, Ising fixed points (8) undergo a pitchfork bifurcation at $\eta=2 \kappa \beta$. At the pitchfork, each Ising LS leads to two solutions with opposite chirality (Bloch LS):

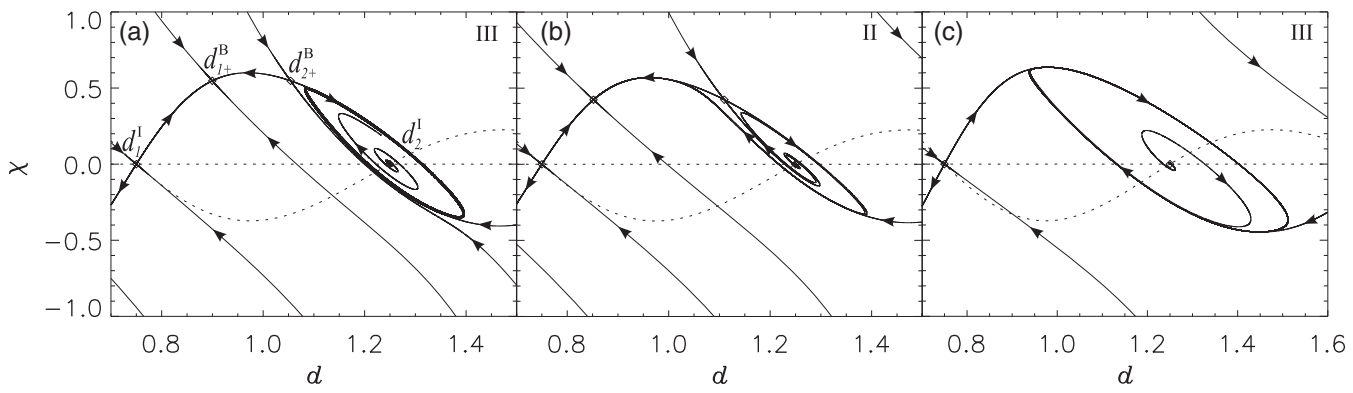

FIG. 5. Phase space of Eq. (7) for (a) $\eta=-1.5$ and $\beta=-3$ (region III), (b) $\eta=-1.5$ and $\beta=-2.8$ (region II), and (c) $\eta=-1.0$ and $\beta=-2.4$ (region III). $\kappa=0.3$. Symbols indicate fixed points and solid lines stable and unstable manifolds. The bold line in (a) corresponds to the limit cycle. The undulating dotted line displays $f(d)$ for reference. 


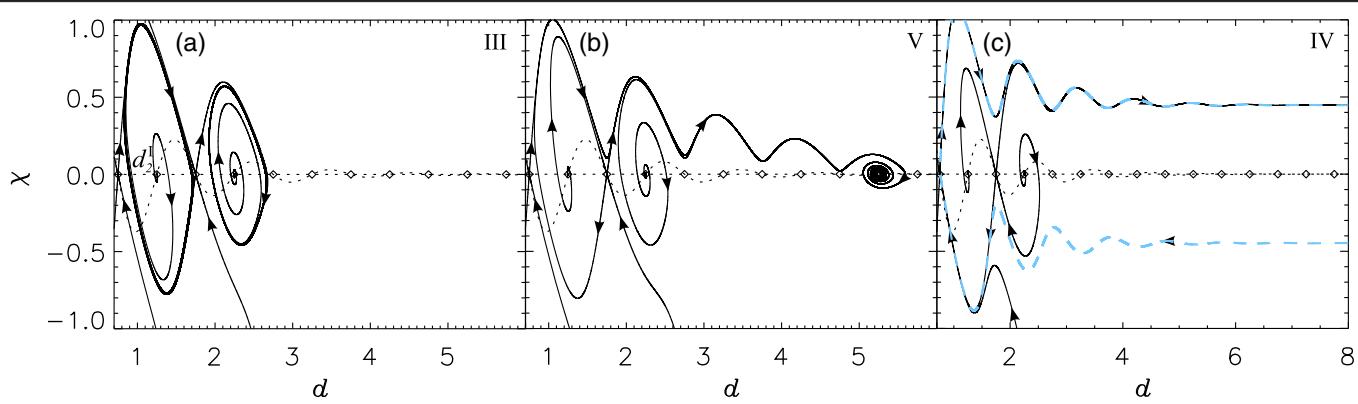

FIG. 6 (color online). As Fig. 5 for (a) $\eta=-0.15$ and $\beta=-3.17$ (region III), (b) $\eta=-0.05$ and $\beta=-3.15$ (region V), and (c) $\eta=0.2$ and $\beta=-3.17$ (region IV). The blue bold dashed line in panel (c) corresponds to a bouncing trajectory of two Bloch walls.

$$
\chi_{ \pm}^{B}= \pm \sqrt{\eta-2 \kappa \beta}, \cos \left(q d_{n \pm}^{B}\right) e^{-d_{n \pm}^{B}}=2 k \chi_{ \pm}^{B} .
$$

Stable Ising LS ( $n$ odd) lead to stable Bloch LS, which are found in the parameter region II delimited by the pitchfork line and the SN-SNIC line to be discussed later. These solutions correspond to the resting Bloch LS of the full model shown in Figs. 1(b) and 1(e). Saddle Ising LS (n even) lead to two saddle Bloch LS, and simultaneously, if $\beta>\beta_{n}^{c}=-A_{n} / 2 \kappa$, where $A_{n}=(-1)^{n} q e^{-d_{n}^{l}}$ the Ising LS becomes stable (region Ib in Fig. 4). Physically, the destabilizing interaction of the tails is counterbalanced by the motion induced by chirality.

These solutions found for $\beta>\beta_{n}^{c}$ undergo a Hopf bifurcation at $\eta=-A_{n}$, leading to the oscillatory regime III. Figure 5(a) shows the limit cycle arising at the Hopf of $d_{2}^{I}$. In the reduced model, oscillations occupy a large region in parameter space, and, in fact, this regime is easily found in the full PDE (Fig. 2). Note also that region II overlaps with $\mathrm{Ib}$ and III. In the overlapping regions several regimes coexist.

Regimes I, II, and III are actually the result of the unfolding of the codimension- 2 bifurcation point $(\eta=2 \kappa \beta$, $\beta=\beta_{n}^{c}$, where the pitchfork and Hopf lines meet. This is a double-zero (DZ) point [46]. The DZ also unfolds a saddleloop (SL) bifurcation $\left(\mathrm{SL}_{1}\right.$ line in Fig. 4). At the SL the cycle originated at an Ising LS with a given even $n$ collides with one of the saddle resting Bloch LS that was born at the pichfork, and it is destroyed. In Fig. 5, panels (a) and (b) show the phase space before and after the collision of the limit cycle originated from $d_{2}^{I}$ with the Bloch $\operatorname{LS} d_{2+}^{\mathrm{B}}$.

The solutions of (9) are the intersection of an exponential with a cosine. Then, changing parameters, solutions with consecutive $n$ collide close to the extrema of the cosine, beyond which there are no Bloch LS. This is a saddlenode $(\mathrm{SN})$ bifurcation that takes place approximately at $\eta=2 \kappa \beta+e^{-2 d_{n}^{ \pm}} / 4 \kappa^{2}$, where $d_{n}^{ \pm}=(2 n+1 \pm 1) \pi / 2 q$ (see Fig. 4). The $\mathrm{SN}$ and $\mathrm{SL}_{1}$ lines collide tangentially at a a second codimension-2 point which is the saddle-node separatrix loop (SNSL). At the other side of the SNSL the saddle-node happens on the circle defined by the cycle; hence, it is a saddle-node in the circle or SNIC bifurcation.
In Fig. 5, panels (b) and (c) show the phase space when crossing the SNIC line from region II to region III.

The oscillatory region III ends at a second saddle-loop ( $\mathrm{SL}_{2}$ in Fig. 4) where the cycle centered at an Ising LS with an even $n$ collides with the Ising LS for $n+1$. Panels (a) and (b) in Fig. 6 show the transition from region III to region $\mathrm{V}$ crossing the $\mathrm{SL}_{2}$ line, where the cycle centered at $d_{2}^{I}$ collides with the saddle $d_{3}^{I}$.

Remarkably the destruction of the limit cycle at $\mathrm{SL}_{2}$ opens an outgoing channel in phase space along its remnants. Two walls initially separated a distance between $d_{2}^{I}$ and $d_{3}^{I}$ move towards each other until reaching a minimal distance close to $d_{1}^{I}$ and bounce back. As they move apart the interaction strength decreases exponentially and, for $\eta<0$ (below the IB transition), walls become of the Ising type and finally stop at an stable equilibrium distance [Fig. 6(b)]. This is regime $\mathrm{V}$ which, despite existing in a relatively small parameter region (see Fig. 4), is also found in the full PDE as shown in Fig. 7.

For $\eta>0$ walls are intrinsically Bloch and after colliding they escape to infinity (regime IV). An incoming channel also opens and Bloch walls initially far away and moving towards each other bounce after reaching a minimal separation [blue bold dashed line in Fig. 6(c)]. The escape trajectory is confined by the unstable manifolds of $d_{1}^{I}$ and $d_{3}^{I}$, which quickly get close due to the stable transverse direction. This regime corresponds to Fig. 3 for the full PDE.
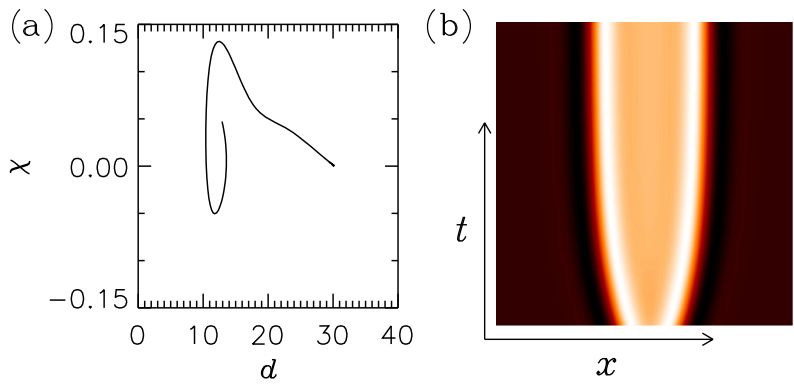

FIG. 7 (color online). Oscillatory Bloch LS in the PCGLE after the cycle has destroyed by changing a parameter. It expands until a large width Ising LS is formed. Here $\gamma=0.32$. Other parameters as in Fig. 2. 
We have derived a simple model for the dynamics of Ising and Bloch walls close to a nonequilibrium IsingBloch transition. It describes the coupled evolution of wall motion, interaction, and chirality. It predicts the formation of Ising and Bloch LS as well as a variety of spatiotemporal behavior including drifting and oscillatory Bloch LS. These results are generic and should thus be observable in different physical systems. In particular, we have reproduced them by numerical simulations of the GinzburgLandau equation and they have also been observed in different physical systems $[17,18]$.

[1] D. Michaelis, U. Peschel, F. Lederer, D. V. Skryabin, and W. J. Firth, Phys. Rev. E 63, 066602 (2001).

[2] L. N. Bulaevski and V. L. Ginzburg, Zh. Eksp. Teor. Fiz. 45, 772 (1963) [Sov. Phys. JETP 18, 530 (1964)].

[3] A.H. Eschenfelder, Magnetic Bubble Technology (Springer-Verlag, Berlin, 1981).

[4] P. Coullet, J. Lega, B. Houchmanzadeh, and J. Lajzerowicz, Phys. Rev. Lett. 65, 1352 (1990).

[5] A. Esteban-Martin, V. B. Taranenko, J. Garcia, G. J. deValcarcel, and E. Roldan, Phys. Rev. Lett. 94, 223903 (2005).

[6] S. Rüdiger, J. Casademunt, and L. Kramer, Phys. Rev. Lett. 99, 028302 (2007).

[7] J. Lajzerowicz and J. J. Niez, J. Phys. Lett. 40, 165 (1979).

[8] M. G. Clerc, S. Coulibaly, and D. Laroze, Physica (Amsterdam) 239D, 72 (2010).

[9] M. Tlidi, A. G. Vladimirov, D. Pieroux, and D. Turaev, Phys. Rev. Lett. 103, 103904 (2009).

[10] M. Tlidi, A. Sonnino, and G. Sonnino, Phys. Rev. E 87, 042918 (2013).

[11] H. Willebrand, M. Or-Guil, M. Schilke, and H.-G. Purwins, Phys. Lett. A 177, 220 (1993).

[12] S. Pirkl, P. Ribiere, and P. Oswald, Liq. Cryst. 13, 413 (1993).

[13] K. L. Lee, W. D. McCormick, Q. Ouyang, and H. Swinney, Science 261, 189 (1993).

[14] P. B. Umbanhowar, F. Melo, and H. L. Swinney, Nature (London) 382, 793 (1996)

[15] M. Pesch, W. Lange, D. Gomila, T. Ackemann, W. J. Firth, and G.-L. Oppo, Phys. Rev. Lett. 99, 153902 (2007).

[16] B. Marts, K. Martinez, and A. L. Lin, Phys. Rev. E 70, 056223 (2004).

[17] F.-J. Niedernostheide, B. S. Kerner, and H. G. Purwins, Phys. Rev. B 46, 7559 (1992).

[18] V. Sánchez-Morcillo, V. Espinosa, I. Pérez-Arjona, F. Silva, G. de Valcárcel, and E. Roldán, Phys. Rev. E 71, 066209 (2005).

[19] I. V. Barashenkov, S. R. Woodford, and E. V. Zemlyanaya, Phys. Rev. E 75, 026604 (2007).

[20] I. V. Barashenkov and S. R. Woodford, Phys. Rev. E 75, 026605 (2007).
[21] I. V. Barashenkov and S. R. Woodford, Phys. Rev. E 71, 026613 (2005).

[22] P. Grelu and N. Akhmediev, Nat. Photonics 6, 84 (2012).

[23] Y. K. Chembo and C. R. Menyuk, Phys. Rev. A 87, 053852 (2013).

[24] S. Coen, H. G. Randle, T. Sylvestre, and M. Erkintalo, Opt. Lett. 38, 37 (2013); S. Coen and M. Erkintalo, Opt. Lett. 38, 1790 (2013).

[25] G. Giacomelli and A. Politi, Phys. Rev. Lett. 76, 2686 (1996).

[26] M. Wolfrum and S. Yanchuk, Phys. Rev. Lett. 96, 220201 (2006).

[27] S. Yanchuk and G. Giacomelli, Phys. Rev. Lett. 112, 174103 (2014).

[28] F. Marino, G. Giacomelli, and S. Barland, Phys. Rev. Lett. 112, 103901 (2014).

[29] P. Coullet, J. Lega, and Y. Pomeau, Europhys. Lett. 15, 221 (1991).

[30] P. Coullet, C. Riera, and C. Tresser, Phys. Rev. Lett. 84, 3069 (2000).

[31] M. G. Clerc, S. Coulibaly, and D. Laroze, Int. J. Bifurcation Chaos Appl. Sci. Eng. 19, 2717 (2009).

[32] P. Coullet, Int. J. Bifurcation Chaos Appl. Sci. Eng. 12, 2445 (2002).

[33] P. V. Paulau, D. Gomila, P. Colet, B. A. Malomed, and W. J. Firth, Phys. Rev. E 84, 036213 (2011).

[34] P. Coullet, C. Elphick, and D. Repaux, Phys. Rev. Lett. 58, 431 (1987).

[35] G.-L. Oppo, A. J. Scroggie, and W. J. Firth, Phys. Rev. E 63, 066209 (2001).

[36] D. Gomila, P. Colet, G.-L. Oppo, and M. San Miguel, Phys. Rev. Lett. 87, 194101 (2001).

[37] T. E. Woolley, R. E. Baker, P. K. Maini, J. L. Aragón, and R. A. Barrio, Phys. Rev. E 82, 051929 (2010).

[38] M. G. Clerc, D. Escaff, and V. M. Kenkre, Phys. Rev. E 72, 056217 (2005).

[39] A. J. Scroggie, J. M. McSloy, and W. J. Firth, Phys. Rev. E 66, 036607 (2002).

[40] L. Spinelli, G. Tissoni, L. A. Lugiato, and M. Brambilla, Phys. Rev. A 66, 023817 (2002).

[41] P. V. Paulau, D. Gomila, P. Colet, M. A. Matías, N. A. Loiko, and W. J. Firth, Phys. Rev. A 80, 023808 (2009).

[42] See Supplemental Material at http://link.aps.org/ supplemental/10.1103/PhysRevLett.114.084101, which includes Refs. [43,44], for the steps leading from the PCGLE to the reduced model (4).

[43] P. Coullet and K. Emilsson, Physica (Amsterdam) 61D, 119 (1992).

[44] A. Yochelis, J. Burke, and E. Knobloch, Phys. Rev. Lett. 97, 254501 (2006).

[45] C. Elphick, A. Hagberg, B. A. Malomed, and E. Meron, Phys. Lett. A 230, 33 (1997).

[46] J. Guckenheimer and P. Holmes, Nonlinear Oscillations, Dynamical Systems, and Bifurcations of Vector Fields (Springer, New York, 1983). 